\title{
Ionisation fronts and their interaction with density fluctuations: implications for reionisation
}

\author{
Ilian T. Iliev ${ }^{1}$, Paul R. Shapiro ${ }^{2}$, Evan Scannapieco ${ }^{3}$, Garrelt \\ Mellema $^{4}$, Marcelo Alvarez ${ }^{2}$, Alejandro C. Raga ${ }^{5}$, and Ue-Li Pen ${ }^{1}$ \\ ${ }^{1}$ Canadian Institute for Theoretical Astrophysics, University of Toronto, 60 St. George Street, \\ Toronto, ON M5S 3H8, Canada, email: iliev@cita.utoronto.ca \\ ${ }^{2}$ Department of Astronomy, University of Texas, Austin, 78712, USA \\ ${ }^{3}$ Kavli Institute for Theoretical Physics, Kohn Hall, UC Santa Barbara, \\ Santa Barbara, CA 93106, USA \\ ${ }^{4}$ ASTRON, P.O. Box 1, NL-7990 AA Dwingeloo, The Netherlands \\ ${ }^{5}$ Instituto de Ciencias Nucleares, Universidad Nacional Autonoma de México (UNAM), \\ Apdo. Postal 70-543, 04510 México, D. F., México
}

\begin{abstract}
The propagation of cosmological ionisation fronts (I-fronts) during reionisation is strongly influenced by small-scale structure. Here we summarise our recent attempts to understand the effect of this small-scale structure. We present high resolution cosmological N-body simulations at high-z $(z>6)$ which resolve a wide range of halo mass, from mini-halos to clusters of large, rare halos. We also study how mini-halos affect I-fronts, through simulations of minihalo photo-evaporation including numerical gas dynamics with radiative transfer. Furthermore, we modify the I-front propagation equations to account for evolving small-scale structure, and incorporate these results into a semi-analytical reionisation model. When intergalactic medium clumping and mini-halo clustering around sources are included, small-scale structure affects reionisation by slowing it down and extending it in time. This helps to explain the observations of the Wilkinson Microwave Anisotropy Probe, which imply an early and extended reionisation epoch. We also study how source clustering affects the evolution and size of $\mathrm{H}$ II regions, finding, in agreement with simulations, that H II regions usually expand, and rarely shrink. Hence, "relic H II regions" are an exception, rather than the rule. When the suppression of small-mass sources in already-ionised regions by Jeans-mass filtering is accounted for, H II regions are smaller, delaying overlap. We also present a new numerical method for radiative transfer which is fast, efficient and easily coupled to hydrodynamics and N-body codes, along with sample tests and applications.
\end{abstract}

\section{Introduction}

When a source of ionising radiation turns on in a neutral gas, the I-front initially moves supersonically as a weak, R-type front and outruns the gas dynamical disturbance it creates (e.g. Spitzer 1978, and references therein). In a uniform gas, the front eventually slows to twice the isothermal sound speed of the ionised gas as it approaches the size of the equilibrium Strömgren sphere and is transformed into a subsonic D-type front, usually preceded by a shock. Thereafter, it is very much affected by gas dynamics. Shapiro \& Giroux (1987) first considered this problem in the cosmological context of the expanding IGM, deriving the general equations describing I-front evolution and solving them analytically for the case of constant gas clumping factor and temperature. They found that global cosmological I-fronts typically stayed in the weak, R-type regime as the density 

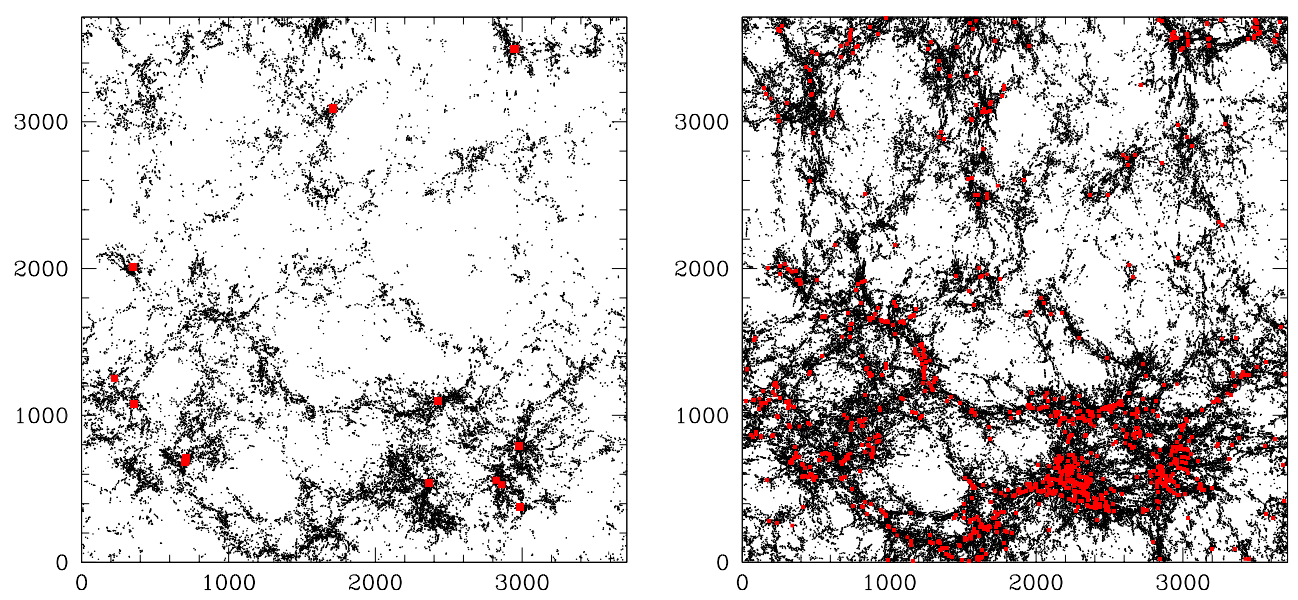

Figure 1. Structure formation at high redshift in $\Lambda$ CDM. Halo distribution at (left) redshift $z=17.2$ and (right) $z=9.42$ from a massively-parallel PMFAST N-body simulation with $1836^{3}$ particles, $3672^{3}$ cells, and simulation box size $10 h^{-1}$ Mpc co-moving. Shown are mini-halos (black dots) and ionising sources (red squares) in a $1 \mathrm{Mpc}$ thick slice (approx. 1/14th of the total simulation volume).

dropped over time, failing to reach the Strömgren radius. This result justifies subsequent approximate treatments of cosmic reionisation in the inhomogeneous IGM that results from structure formation which neglect the gas dynamical back reaction of the I-fronts. However, when the I-fronts encounter self-shielding inhomogeneities (like halos) large and dense enough to trap the I-fronts and convert them to D-type, this approximation must break down.

\section{Structure formation at high redshift and its effects on I-front propagation during reionisation}

Within the CDM paradigm cosmological structures form hierarchically in time, starting with the smallest-scale structures at the highest redshifts, which grow through accretion and mergers to form larger and larger structures. While the cold dark matter clumps gravitationally on all scales, only virialised halos with total mass above the cosmological Jeans mass can retain their gas content. These virialised halos can be divided into two main classes, halos with virial temperature above $\sim 10^{4} \mathrm{~K}$ whose gas can cool through atomic line cooling of hydrogen, leading to efficient star formation in such halos, and smaller-mass halos (mini-halos) whose gas in absence of metals can only cool through $\mathrm{H}_{2}$ molecules, which are fragile and easily destroyed. Thus, the first class of halos are generally believed to be the dominant sources of the radiation responsible for cosmic reionisation, while typical mini-halos were unable to form stars once the first generation created a background of dissociating UV starlight. Subsequently, mini-halos were just inert balls of neutral gas. Fig. 1 shows the halos which formed in a large N-body simulation of early structure formation. The larger (source) halos (red squares) are quite rare and highly clustered, especially at high redshift, as expected for rare density peaks in a Gaussian density field. On the other hand, the mini-halos (black dots) are quite common at all times and are strongly clustered around the ionising sources, covering their sky (Shapiro 2001). 

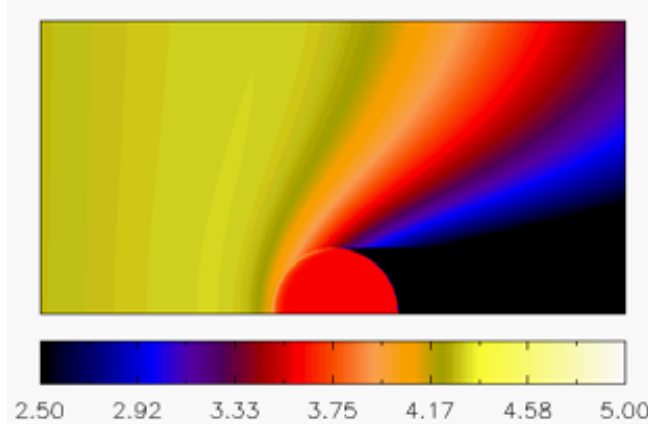
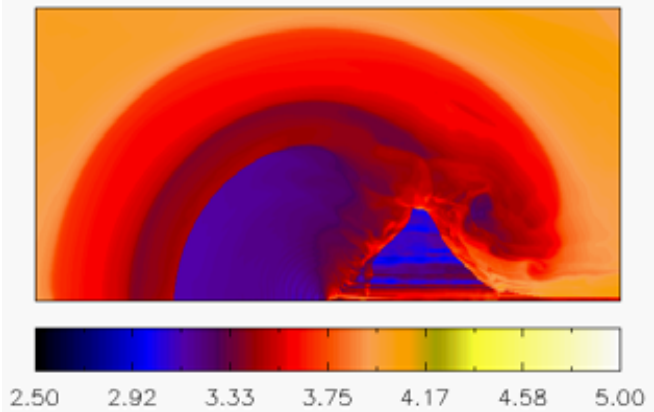

Figure 2. Photo-evaporating mini-halo snapshots of temperature for a $10^{7} M_{\odot}$ mini-halo encountered by a global I-front at $z=9$ from a source with a $50,000 \mathrm{~K}$ black-body spectrum. The times shown are: (left) $0.2 \mathrm{Myr}$, when the I-front is still fast, R-type, with extended structure in the IGM due to the finite photon mean free path clearly visible, and (right) $150 \mathrm{Myr}$, close to complete evaporation, after which only a dark halo devoid of gas remains. Note the complex flow structure which involves multiple shocks. Shaded isocontours indicate the values of $\log _{10}(T)$, as labelled on the colour bar.

I-fronts propagating outward from the ionising sources encounter these mini-halos and get trapped inside them, eventually completely photo-evaporating all the gas. We have studied this process in detail, by means of high-resolution adaptive-mesh-refinement (AMR) hydrodynamic and radiative transfer simulations (Shapiro et al. 2004; Iliev et al. 2005d). In Fig. 2 we show two snapshots of the photo-evaporating flow's temperature structure at times $t=0.2 \mathrm{Myr}$ (during the fast, R-type phase), showing the relatively extended I-front structure compared to the mini-halo size, and $t=150 \mathrm{Myr}$, close to the moment of complete evaporation of the mini-halo, which shows the complicated structure of the photo-evaporating flow, with multiple shocks (see papers for details). We have performed a large number of these high-resolution simulations (with finest grid up to $(r, z)=1024 \times 2048$ in 2 -D axisymmetry) and found simple analytical fits to the total consumption of ionising photons and evaporation times as functions of the mini-halo initial mass, redshift, and the flux and spectrum of the ionising source (Iliev et al. 2005d). We showed that mini-halos can increase the global ionising photon consumption during reionisation by a factor of up to 2 compared to the mean IGM alone.

Using our fitting formulae to our detailed simulation results, we were able to modify the I-front propagation equations of Shapiro \& Giroux (1987) to properly reflect the increased consumption of ionising photons due to the mini-halos and the corresponding slowing down of the global I-fronts (Iliev et al. 2005c). We included the effects of infall and mini-halo bias around the sources, the evolving mean IGM clumping and the dependence on the photon production efficiencies, spectra, and lifetimes of the ionising sources. We found that small-scale structures have a significant effect on the progress of reionisation, slowing it down, and extending it in time, which can help us understand the recent observations by the Wilkinson Microwave Anisotropy Probe satellite, which point to an early and extended reionisation epoch (Fig. 3). More recently (work in progress) we extended our formalism to account for the clustering of the ionising sources. We find that the clustering of source halos in time and space leads to multiple sources in each $\mathrm{H}$ II region, which dramatically changes the numbers and sizes of the ionised regions and hence the overall topology of reionisation (Fig. 4 and Fig. 5, left). H II regions around short-lived individual sources are small (less than $1 \mathrm{Mpc}$ co-moving around the smaller sources) and quickly recombine and shrink in size once the source ends its life, forming socalled "relic H II regions", but when source halo clustering is taken into account the H II 

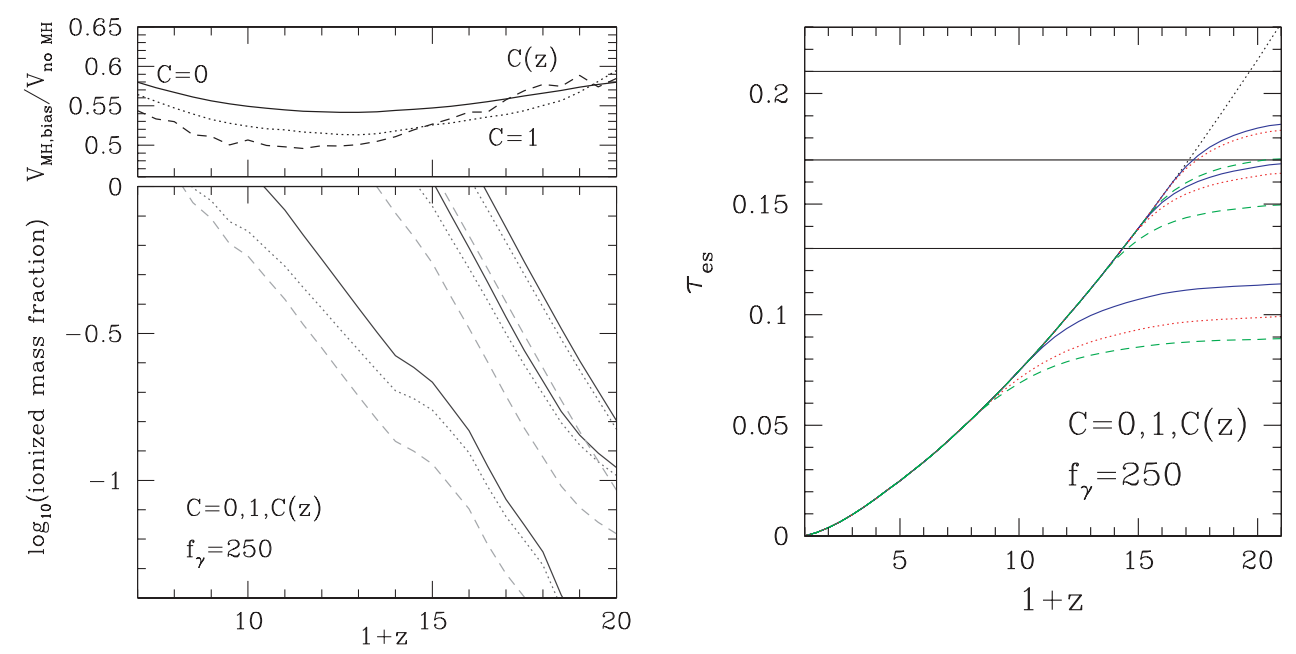

Figure 3. Global reionisation. Sources with $f_{\gamma}=250$ and a lifetime of $t_{s}=3 \mathrm{Myr}$ are assumed. (bottom panel) Decimal logarithm of ionised mass (or Lagrangian volume) fractions (i.e. 0 corresponds to overlap) for the cases of no mini-halos (solid), unbiased mini-halos (dotted), and biased mini-halos (dashed) for IGM clumping factors (top to bottom in each case) $C=0,1$, and $C(z)$ (clumped IGM). (upper panel) Ratios of the ionised volume fractions in the presence of biased mini-halos and with no mini-halos for $C=0,1, C(z)$.

regions expand continuously, reaching sizes of up to tens and hundreds of co-moving Mpc. In that case, relic H II regions do not generally form. We also studied the consequences of the suppression of the formation of smaller sources inside the already-ionised regions (due to increased Jeans mass there, in correspondence to the higher gas temperature of $\sim$ few $\times 10^{4} \mathrm{~K}$ ) (Fig. 5, right). We find that sufficiently strong suppression can have quite dramatic effects, decreasing (in the particular high-redshift source case we consider here) the size of the H II region by an order of magnitude, and leading to a temporary formation of a small relic H II region. However, for lower-redshift sources this suppression has much smaller consequences since the larger ionising sources (whose formation is not affected by this suppression), which were very rare at high-z, become more abundant and start playing a more important role in the process of reionisation.

\section{A new photon-conserving method for transfer of ionising radiation}

In the last few years there has been an intense development of new numerical radiative transfer methods for cosmology (e.g. Abel et al. 1999; Gnedin \& Abel 2001; Razoumov et al. 2002; Maselli et al. 2003). However, most of the existing methods are not efficient enough to be directly coupled to gas- and N-body dynamics, which limits the scope of their applications. One common problem is that the fast, R-type I-front propagation timescale (sometimes approaching the light-crossing time) is much shorter than the fluid dynamical and gravitational times, leading to the necessity of very small time-steps to track the I-fronts correctly. In addition, the bound-free opacity of the neutral gas is very high, so algorithms for spatial differencing the transfer equation often require very small cell sizes, and this implies very small time steps even when the I-fronts are subsonic. Our photo-evaporation simulations described above (Shapiro et al. 2004; Iliev et al. 2005d) utilised an AMR Eulerian hydro code in 2-D axisymmetry, capable of handling a large range of scales with high resolution efficiently enough that we were able to take very small 

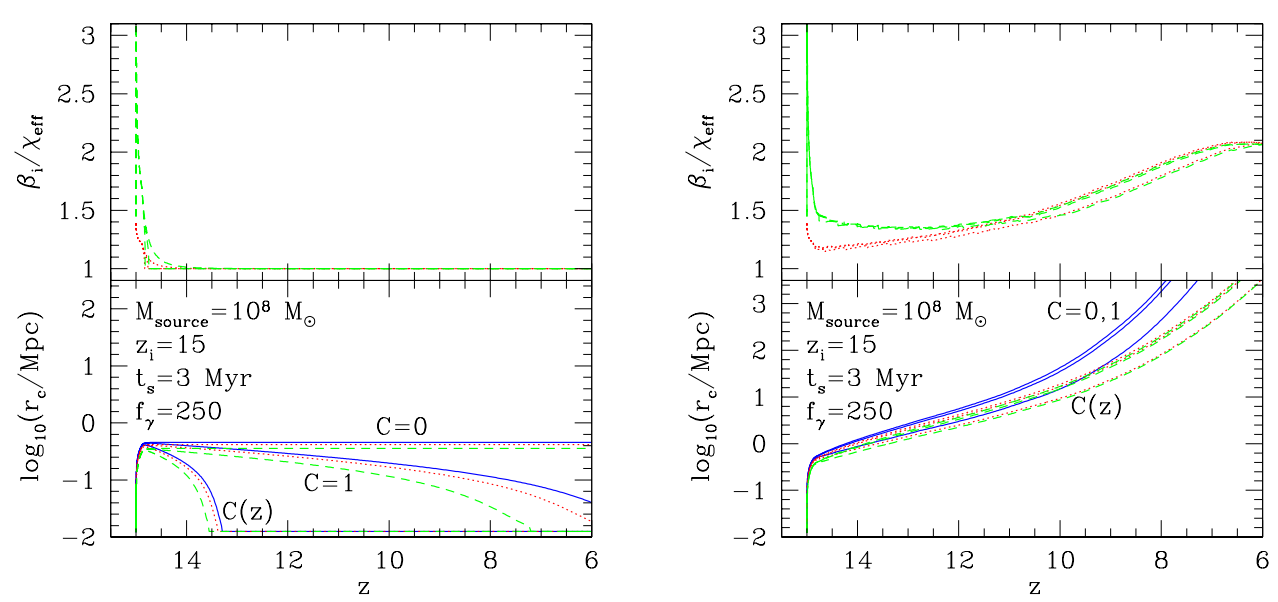

Figure 4. Evolution of an individual H II region about a central source of mass $10^{8} M_{\odot}$ which turns on at redshift $z=15$, for (left) a single source, and (right) clustered multiple sources for $C=0,1, C(z)$. Top: the correction factor $\beta_{i} / \chi_{\text {eff }}$ due to mini-halos for the number of ionised photons consumed per atom that crosses the I-front, for biased (dashed) and unbiased mini-halos (dotted). Bottom: co-moving radius of the H II region for no mini-halos (solid), unbiased (dotted) and biased mini-halos (dashed).
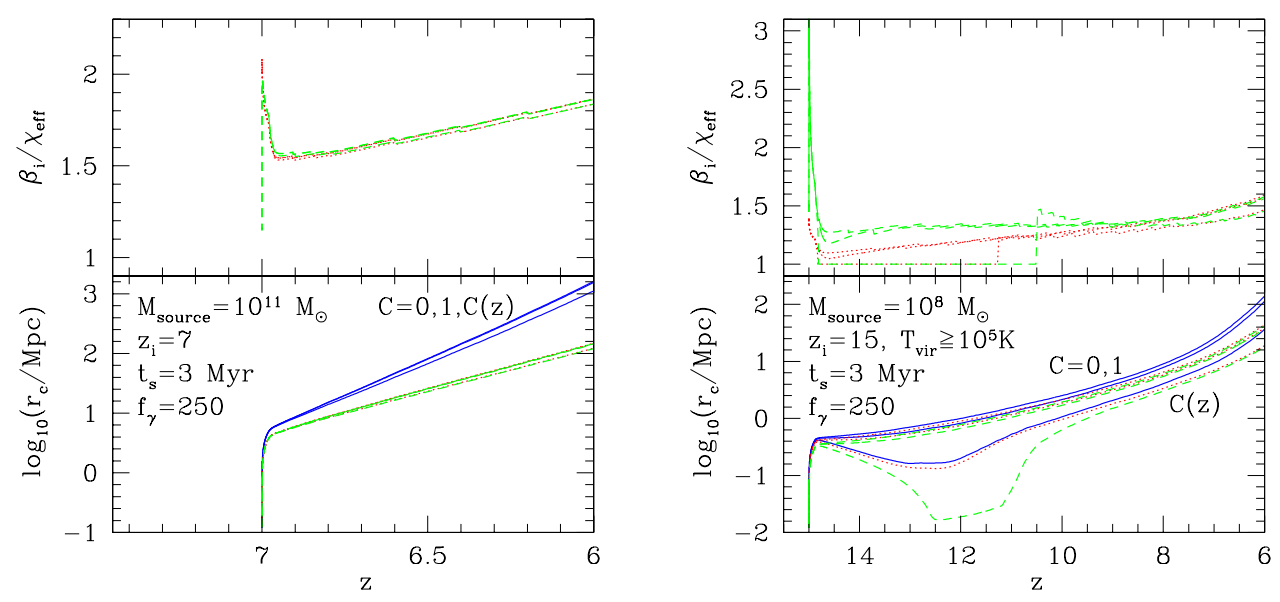

Figure 5. Same as Fig. 4, for clustered sources, but (left) a central source of mass $10^{11} M_{\odot}$ turning on at $z=7$, and (right) assuming that the formation of ionising sources with virial temperatures below $10^{5} \mathrm{~K}$ is suppressed within the H II region.

time-steps and very small cell sizes where needed. However, to generalise these "zoomedin" calculations to 3-D and a simulation volume large enough to encompass both smalland large-scale structure, it is necessary to develop a more sophisticated radiative transfer algorithm. We have recently developed such a method, which accurately follows both fast and slow I-fronts without need to adopt such small time-steps or cell sizes. We achieved this by matching the ionisation rates and flux attenuation along rays to ensure photon conservation, and time-averaging the neutral column densities per cell per step to give the correct I-front position and speed even for large time-steps and cell-widths (Mellema et al. 2005; Iliev et al. 2005b) (see papers for details). These characteristics make our 

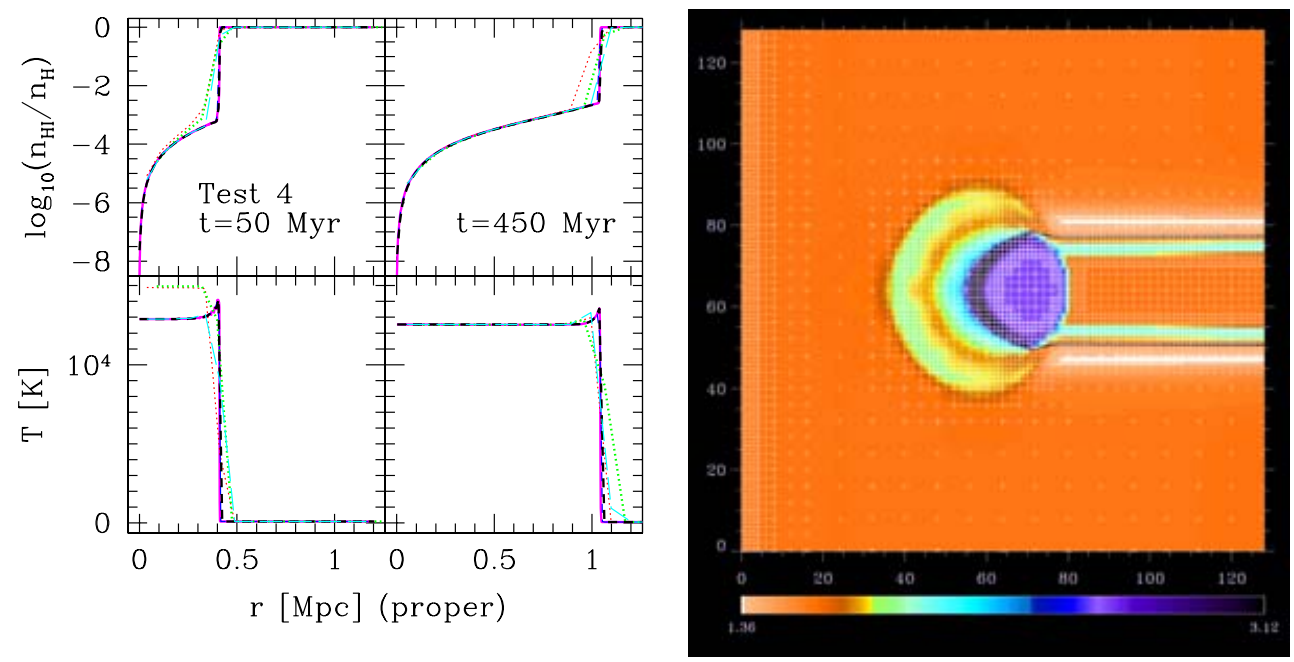

Figure 6. Photon-conserving RT. (left) Neutral hydrogen (top) and temperature (bottom) profiles for a cosmological I-front starting at $z=9$. The left panels show time $t=50$ Myrs after turn-on (= 1 time-step in the coarse temporal resolution case, and 10 time-steps in the high temporal resolution case), while right panels show the solution at $t=450$ Myrs $\left(=3.5 t_{\text {rec }}\right)$. Thin lines correspond to $1-\mathrm{D}$ results with 16 cells, 10 time-steps (dotted, red); 16 cells, 100 time-steps (long-dashed, cyan); and 128 cells, 100 time-steps (short-dashed, blue). Thick lines correspond to $3-\mathrm{D}$ results with $32^{3}$ cells, 10 time-steps (dotted, green) and $256^{3}$ cells, 100 time-steps (short-dashed, black). Finally, the reference "exact" solution (1-D, 1024 cells, 100 time-steps) is shown by thick, solid, magenta line. (right) A 3-D, AMR gas dynamics and radiative transfer simulation of the photo evaporation of a dense gas clump calculated with a 5-level adaptive mesh. The snapshot shows $\log _{10}(n)$, gas number density in the central $x y$ plane. White crosses indicate the computational mesh, while black shows the I-front position.

method very efficient for direct coupling with gas dynamic and N-body codes. We have tested the method in detail, on both fixed and adaptive grids, as a stand-alone radiative transfer code as well as coupled to AMR gas dynamics (for some sample results see Fig. 6).

\section{References}

Abel, T., Norman, M. L., Madau, P., 1999, ApJ, 523, 66

Gnedin, N. Y., Abel, T., 2001, New Astr., 6, 437

Iliev, I. T., Alvarez, M., Scannapieco, E., Shapiro, P. R., 2005a, in preparation

Iliev, I. T., Mellema, G., Shapiro, P. R., 2005b, in preparation

Iliev, I. T., Scannapieco, E., Shapiro, P. R., 2005c, ApJ, in press, (astro-ph/0411035)

Iliev, I. T., Shapiro, P. R., Raga, A. C., 2005d, MNRAS, in press, (astro-ph/0408408)

Maselli, A., Ferrara, A., Ciardi, B., 2003, MNRAS, 345, 379

Mellema, G., Iliev, I. T., Alvarez, M., Shapiro, P. R., 2005, in preparation

Razoumov, A. O., Norman, M. L., Abel, T., Scott, D., 2002, ApJ, 572, 695

Shapiro, P. R., 2001, in AIP Conf. Proc. 586: 20th Texas Symposium on Relativistic Astrophysics, p. 219

Shapiro, P. R., Giroux, M. L., 1987, ApJ, 321, L107

Shapiro, P. R., Iliev, I. T., Raga, A. C., 2004, MNRAS, 348, 753

Spitzer, L., 1978, Physical processes in the interstellar medium (New York Wiley-Interscience, 1978) 